INTERNATIONAL DESIGN CONFERENCE - DESIGN 2018

https://doi.org/10.21278/idc.2018.0179

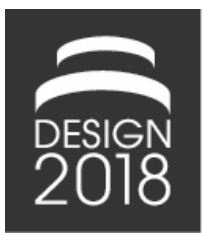

\title{
INTRODUCING AN EVALUATION FRAMEWORK FOR WEARABLE DEVICES DESIGN: EXPLAIN REASONS OF LOW USER ADOPTION
}

\author{
Y. Wang, S. Yu, J. Wang, N. Ma and Z. Liu
}

\begin{abstract}
We summed up the factors and attributes affecting the success of smart wearable product design. We analysed the relations between design intention and cognitive interpretation and presented the attributes of aesthetic experience and emotional expression in wearable device. Then we propose an evaluation framework for better understanding the attributes, impact factors of successful wearable devices design. The evaluation framework can explain the reasons for low user stickiness of wearable device, and the framework can be used for determining design directions in the concept phrase of design.
\end{abstract}

Keywords: user requirement, user experience, aesthetics, design cognition, user scenario

\section{Introduction}

With the rapid development of big data, wearable computing and wireless communication technology, the positioning of wearable devices has gradually transferred from providing personal services into data driven social services and being an important entrance of Internet of things. Taking fashion decoration, game and entertainment, health care and other typical application scenarios as the breakthrough point, wearable products burst into market in the past five years (Ananthanarayan and Siek, 2012). Despite the emergence of a large number of wearable smart devices, no much more wearable devices actually being found and popular on the street because of low user adoption (Kobayashi et al., 2009). Just like Donald A. Norman pointed out: "all new technologies will take a while for us to figure out the best manner of interaction as well as the standardization that removes one source of potential confusion" (Norman, 2010). In fact, wearable devices to truly integrate into our daily lives, there is still a long way to go. Nevertheless, due to the inevitable trend of intelligent and digital and continued interest in wearable technology, a large amount of smart wearable equipment is still being designed and produced on a global scale (Chung et al., 2013). While current dealing with the limited sensing capabilities as well as limited fashion possibilities offered by the devices themselves, wearable devices design can augment these possibilities.

The nature of wearing on the body with complex sensors, wearable devices face more challenges coming from design and production. Comfort, safety, durability, convenience of interaction, the effectiveness of information transmission requirements should be considered with commercial strategy in the whole process of design and production, so these challenges made designers face more design constraints and decision-making trade-offs than ever before. In wearable product design practices, there are an increasing number of intelligent wearable hardware firms attempted to use product form design elements with sense of technology and fashion to catch users' attention and transform them into buyers (Yoon et al., 2016). However, it is easy for designers to show a "wow" wearable devices to the 
customers in the first time they saw it, but difficult to ensure the continuous use of the product and keep adoption, which may even have a negative impact on brand communication and promotion.

Smart wearable devices are traditionally associated with functionality and utility of needs, whereas fashionable products deal more with aesthetic, symbolic, and cultural meanings, as well as our emotional needs as individual and social beings. As special form of technology and fashion, wearable products should consider all the aspects mentioned above. There has been a wide range of papers about wearable computing, sensing technology, usability testing of interface and other aspects about how to design strong sensing and friendly smart devices (Yoon et al., 2016). However, few methods and little guidance can be found in design research literature about how to evaluation the wearable devices design in a macroscopic perspective, not merely limited to product design, interaction usability, aesthetic evaluation and function realization etc.

This research focuses on how to evaluate the wearable product design to gain continuous usability (Hallnäs and Redström, 2001). In this paper we review the difficulties we faced in wearable devices design and discussed the relevant aspects of different user scenarios of wearable devices and cognition gaps between designers and users. We presented an evaluation framework by identifying wearable product design attributes and impact factors to understand user perceived value and their design directions for the promotion of sustainable use. This paper is not a conflict to interaction usability or aesthetic evaluation, but a complement and further guidance for smart hardware companies to deal with problems of low user adoption of their products.

This paper addresses how to evaluate wearable devices design to meet diverse and dynamic user requirement in the mobile internet era. This topic is explored in more detail through the following research questions:

- RQ1: Are there cognitive difference and gaps between users and designers toward to wearable devices, and how to overcome these problems?

- RQ2: What are the factors that affect the users' acceptance of wearable devices?

- RQ3: How to improve the user adoption for using smart wearable devices?

It is important to maintain the cognition consistency of users and designers while designing the wearable devices (Da Silva et al., 2015). Besides that, identifying the affecting factors of users' acceptance of wearable devices is also very important to gain sustainable competitiveness. Meanwhile, these questions reflect an improvement from seeking to understand present conditions and their genesis, towards an evaluation framework focus on wearable devices design in the dynamic environment. In the following sections, I outline the methodological means through which I address the stated research questions.

\section{Understanding cognitive gaps between designers and users}

\subsection{User motivation, behaviour and perceived value}

Generally speaking, design is understood as a creation of objects, that is, design for developing new product. But in terms of its essence, design is just to achieve some kind of experience, through which to create some kinds of "behaviour", this process also needs product, but only as a medium to achieve the act. User behaviour is composed of a series of actions, each of which is associated with a target, and the target is the overall goal of motivation (Sohn and Nam, 2015). Conscious action is driven by a series of unconscious operations, which are defined as the customary response conditions during the execution of the action. The implementation of a specific action needs to carry out a conscious plan, arrangement and decision. When a small operation is performed, the user does not need to make a plan in advance because the user is familiar with it. Motivation is an intrinsic condition for the behaviour of an individual. It can't be externally assigned, but can be stimulated or changed. Trigger factors have influence on user's motivation, as an external condition, it can be captured and used by the designer. The occurrence of user behaviour and decision change is mainly related to motivation and trigger factors. However, designers can't directly contact with the product users, so it is difficult to enhance the user's ability through practical training, and high cost of learning products for users is not friendly enough and with poor usability. 
User motivations determine user behaviour while influencing perceived value of the product (Zhou et al., 2011), as shown in Figure 1, user perceived value is the perception of the actual effect of the product attributes that are used to promote or hinder the realization of their goals and objectives, as well as the perceived preference and evaluation results of use of the product Woodruff combined the properties and performance of the product with the customer's specific scenario, and considers that the result of the product is directly related to the user's goal and intention (Woodruff, 1997). Once there is a change in the use of the scenario, the attributes of the product, the results of the use, and the expectations of the customer will change accordingly. Meanwhile, the motivation for users' behaviour of buying or keep wearing on the wearable devices and other tech-fashion products is totally different (Cao et al., 2013). For example, if a college student needed a wearable watch but he didn't have enough money, but one day he find one with nice appearance and right price, he bought it driven probably by the motivation of price and shape, or the basic function of this smart watch. However, if he didn't want to use it after two months, the motivation may lies in that he's perceived value is lower than the cost and resulting in a sense of boredom. Accordingly, the perceived value changed the attitude and behaviour of user.

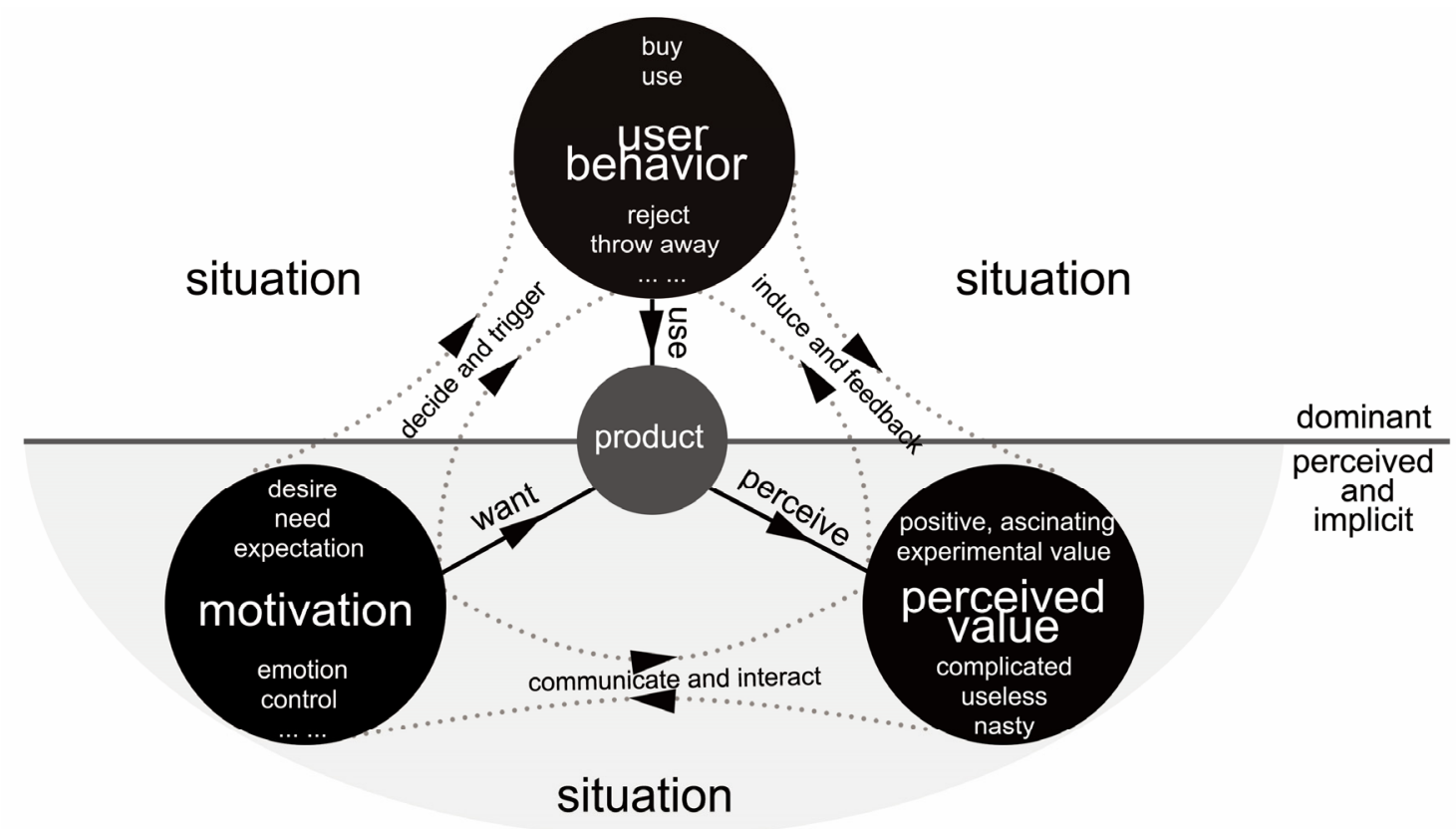

Figure 1. Relationships among user motivation, behaviour and perceived value

\subsection{Design intention and cognitive interpretation}

The understanding of the links between design intention and user cognitive interpretation of the product is still low, especially in a new design area, including wearable product design. The difficulty lies in the fact that the user's feeling of a new product is a more complex cognitive process and many intricate factors contribute to the perception mechanisms (Davis, 2010). With some asymmetry in the design information, designers and users, as the two sides of information exchange, are not only the own unique cognitive subjects, but the co-creator of meaning achieved through communication. It is difficult for designer to completely obtain the design knowledge from the users, even using the method of user participation or empathy into design process (Carroll, 2002). Furthermore, user perception established links different kinds of variables, relative to the assessment of object which controlled by the subject's perceptions; the "design elements", represented by the physical characteristics which define the product by the designer. Although the concept and expression of the design takes into account the needs of the user, the understanding and conveying of the designer is still individual, and the final design is not merely the simple replication of the user desire. A good design should exceed the user's expectations, create and guide the user's requirements. Meanwhile, product 
design is a media of exchange and expression of design information, designers express the design intent through the product, and the users take the initiative to accept and produce an independent interpretation, shown in Figure 2.Therefore, the effectiveness and reliability of design intention benefit the performance of the product design to satisfy user needs interpreted by their perception.

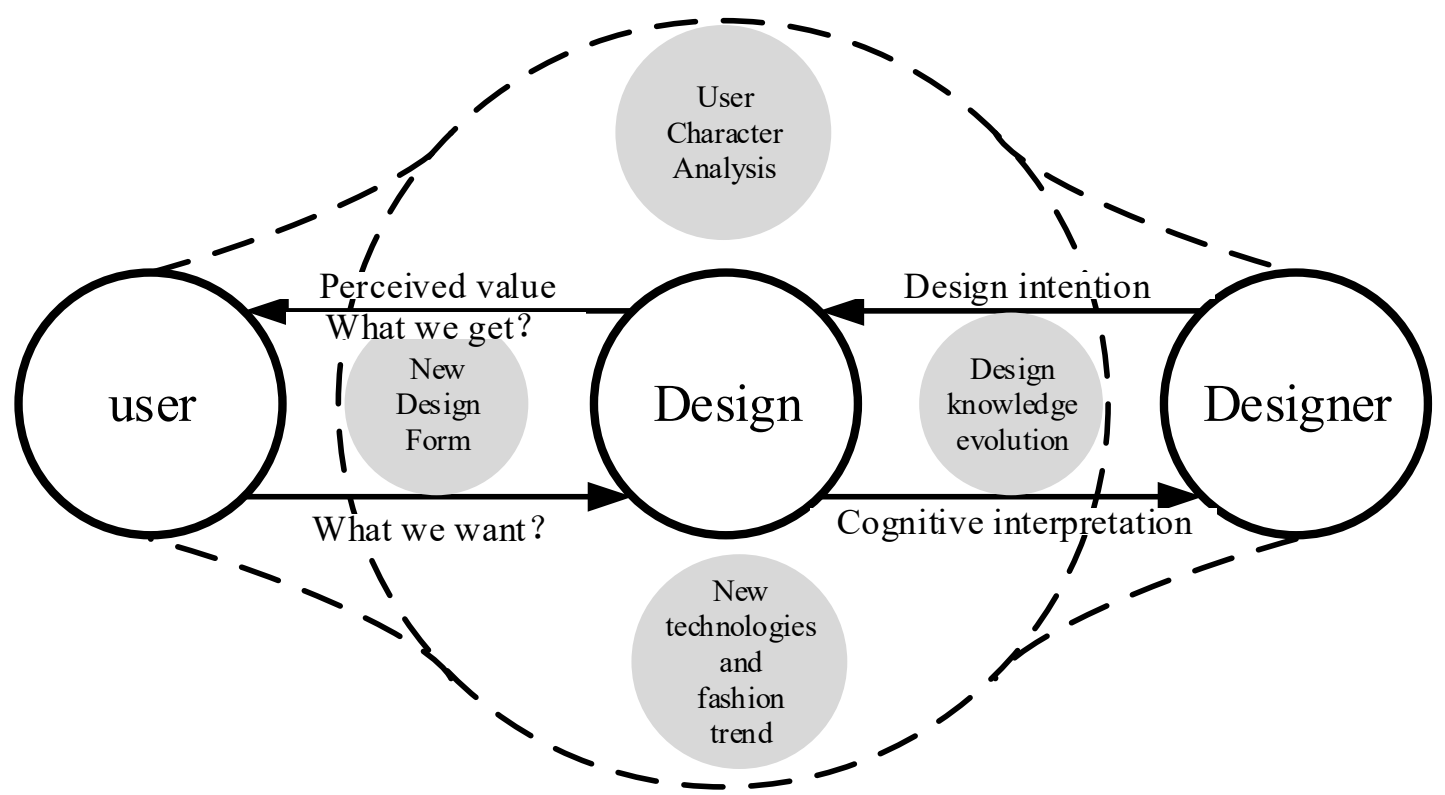

Figure 2. Design intention and cognitive interpretation between user and designer

Design intention need to be identified and interpreted by the user (Deckers et al., 2011). User cognitive interpretation is stimulus identification and interpretation feedback based on the visual, tactile, auditory, olfactory and taste. In wearable products design activities, the design intention has structural cognitive elements (fabric, lighting, stylish, shape and graphics), through the styling and semantic realization of the design intention of materialization and conceptualization, getting the wearable device entity with style and brand. Based on visual perception and style expression, users identify and understand the wearable design through a certain cognitive path according to individual knowledge and experience to shape specific cognitive patterns. Wearable products design is the form and meaning of the tech and fashion to create a unique style attributes, brand attributes and product culture, wearable smart product styling should have a good understanding and communication.

\section{Dynamic user requirement and diverse $\mathrm{UX}$ in wearable design}

\subsection{Dynamic requirement in wearable product design}

User requirement is an important factor to drive product design evolution. In order to get higher user satisfaction and achieve better user experience, designers should give full consideration to the aspects in the effective acquirement and analysis for user requirements and technical requirements (Wang and $\mathrm{Yu}, 2016)$. In wearable industry, it is difficult for designer to get the real needs of users because the dynamic characteristics of requirements in different times. When the customer said he want a cool and fashionable devices, maybe he just talking about it, and what he really want is merely a toy that plays only one time.

Understanding user requirements needs insight details in life and specific usage context, while there's a gap and inconsistency between designers and users in design requirement recognition. Meanwhile, user in different market segments proposed diversified requirements in different user clusters. A product contains different kinds of requirements which have different weight (Wang et al., 2017). In other words, they are ranked in order but the order is varying with time. Moreover, there are some differences in users' professional degree and daily usage habits, then the user requirements tend to be 
ambiguous, even some users don't know what they want. Furthermore, with the emergence of new materials and new technologies applying in wearable products, user requirements for new product are becoming various, and the changing speed is fast. Such as the HoloLens virtual reality glasses, developed by Microsoft, bring users new and robust requirements.

\subsection{Diverse user experiences of wearable smart devices}

The User experience (UX) is largely influenced by user characteristics (physical and mental), user scenario and user interfaces. The ISO 9241-210 stressed that the design addresses the whole user experience, and defined UX as not only a consequence of the presentation, functionality, system performance, interactive behaviour and assistive capabilities of interactive system, both hardware and software, but a consequence of the user's prior experience, attitudes, skills, habits and personality. User experience, to some extent, determines the quality of the products or the services, having a great influence on brand loyalty. However, experiences with products are to be ascribed in part to the products, as the remaining part is due to the context in which the interaction occur and to the users themselves.

In other words, interaction produce experiences, and the way of human-product interaction affect product experience in the whole process of using product. The ultimate goal of product design is to satisfy some requirements or to obtain some kind of services through the human-product interaction, including aspects of tangible interaction and the invisible emotional exchange, both from the objective and subjective perspectives, and internal external aspects. However, in order to adapt to the trend of diversification and multi-channel interaction, human-product interaction has developed from a conventional graphical user interface to a more intuitive, natural and adaptive interactive interface technology, perception user interface technology such as voice interaction, eye-movement tracking, gesture control, virtual reality and brain-computer interface technology makes this natural interactive mode come true.

The innovation of interactive technology brings a new experience to the users, and the users gradually formed a new attitude and habits, as well as new skills and personality cognition towards the product. UX is dynamic, always evolving, scalable, cumulative, and provisional; it develops over time and, as it does, it enriches permanently (Wang and $\mathrm{Yu}, 2016$ ). There is a transition from the devices not nice to have to need to have and bring good experience for health care and sport monitoring, as this products being a permanent part of our lives. Then the wearable VR and AR devices bring users a new game experience which never happened before.

\subsection{Aesthetic experience and emotional expression}

Aesthetic experience is one of the most important but also one of the vaguest and most poorly specified concepts in the psychology of art and design aesthetics (Xenakis and Arnellos, 2013). The processing of aesthetic information for human is based on cognitive structures which are capable of dealing with semantically and perceptually demanding tasks, such as the interpretation of multi-level symbolism, association of distant narrative frameworks into temporally and conceptually coherent structures, detection of sophisticated compositional regularities, integration of symbolic, affective information and multi-level perceptual and so on. Successful realization of such complex mental activities requires high concentration and awareness and efficient working memory processing. Aesthetic emotions could be identified as one of kinds of pleasures of the mind, which have no distinctive physiological and behavioural expressions typical for basic emotions and pleasures of the body. Namely, pleasures of the mind are not simple emotional reactions, but rather collections of emotions distributed over time.

Aesthetics and fashion become very important factors to decide whether to buy the smart products for consumers. In wearable devices design, material, user interface, lighting and other decoration are significant design expressions relating to aesthetic experience, which can cause emotion changes of human product interaction (Wang and $\mathrm{Yu}, 2017$ ). Taking the user's emotional demands and psychological appeal as important design factors to be considered, designer can make it possible for users to always have a good mood in the whole process of interaction between human and smart devices. Only the users have a pleasant, positive, rich emotional experience until forming a strong 
resonance, can the fashionable products enable consumers to produce a unique way of thinking and behaviour, and even to produce a kind of cultural and psychological long-term trust and attachment on the products or services. Furthermore, by satisfying the user's curiosity, stimulating the desire of the user to explore and use, the interest of the interactive process of emotion, which is the basis for the establishment of a good user experience, becomes the driving force of the user's continuous interaction. Wearable smart product design can enhance the user experience of the degree of activity, adhesion and memory with high emotional dependence.

\section{Scenario trigger and implicit interaction}

\subsection{User scenario triggers user requirement and experience}

Scenario is an ongoing story about human and their behaviour. Alvin Toffler defines the scenario as five parts, the environment, the situation, the role, the concept and the information in his book "Future shock" in 1970, and he believed that people have a cognitive process of the specific scenario, so we can make the prediction of cognitive logic behaviour according to the cognitive results (Toffler, 1970). User scenario refers to the relevant information of the user and the surrounding environment when using relevant life experience and aesthetic experience in the process of experience and product cognition (product information decoding), including the user's basic information, the user's surrounding environment information and the user's interaction information feedback by product (Bødker, 2000). The user's perception and cognition of the scenario, which will have a decisive impact on the interactive behaviour of user, is the process of understanding and processing of scenario information in product experience.

User scenarios trigger true user requirement. The real user requirements only appear in the determined user scenario, accordingly, the functions we design in product to meet the user needs should under the specific scenario. So designer can use the user scenarios of product to judge and determine whether a real demand can be used on it. Depth consideration of how the user is used to meet the needs of the product, but also needs to be user needs into the user scene. Only reduce the product into the original user scenarios, designer can give full consideration to the user requirement that the product needs to meet and produce a good user experience. However, there is a distinction of strong or weak and frequency or low frequency of user scenarios, so from the vitality of the scene itself, we sum it up into three dimensions: frequency, duration, and substitutability. High frequency, long duration, irreplaceable user scenario is the important reference for designer to determine the user requirements.

Scenario is the premise of user experience. UX is conscious because the user is aware of what he or she is living, feeling, and sensing, as a result of the interaction with an artefact in specific scenario. While the user behaviour in the process of experience is unconscious and spontaneous based on the user scenario. The key to achieve a good UX is not just increasing or decreasing the content to adapt to the typical target scenarios, but in accordance with specific scenarios by gradual adjustment of experience mode. Furthermore, as an important design basis for designers, user scenario, is not only the carrier of design, but also a significant factor to affect the success or failure of UX and have an impact on design decision, design thinking expression and design behaviour generation. Meanwhile, user's perception of the scenario, as well as cognitive behaviour, has a crucial role in the context of experience design, can help designers to analyse UX at the level of interaction behaviour.

\subsection{Implicit interaction in wearable devices}

Context awareness technology, relying on sensor data to perceive the information and other data to understand the user's situation, location, states and intention so as to better help the user to complete the work schedule, to bring the perfect experience of intelligent life. Mobile context awareness computing can obtain information on the situation and learn the user characteristics, hobbies, habits, health condition and other information from mobile smart devices and provide services information of health, position, reminders, traffic guidance, remote condition and commodity recommendation to satisfy user requirements. Implicit interaction is defined as "an action, performed by the user that is not primarily aimed to interact with a computerized system but which such a system understands as 
input" and which happens "outside of the user's notice or initiative" (Chu, 2009). Relying on contextawareness computing, implicit interaction is occurs when users interact with a product or intelligent system in a determinable but subconscious manner, without the user being aware of it (Wei et al., 2014). It has been harnessed in a number of interactions, such as user-interface interaction and smart home interaction systems.

In wearable interactions, implicit interaction not only has the advantage of real-time access to human body and environmental information by wearing smart devices, but also can make up for the disadvantages of some devices that are not easy to be explicitly touched. Users can focus their attention on the natural behaviour of everyday life, rather than on the input of the interface with high attention. Therefore, the results of implicit interaction feedback should be based on the background information to reduce the interference of the normal activities of the users. Meanwhile, because of the diverse situations and ambiguity of intention judgment of the interaction, it's not suitable for implicit interaction to be applied in the judgment of critical operation to avoid frequent or irreversible misoperation. Moreover, the implicit interaction depends on the user scenarios, if the user is in walking or running situation, it's not appropriate that swing arm to map the interactive operations of wrist wearable devices.

\section{Introducing the evaluation framework for wearable devices design}

\subsection{Goals of the evaluation framework}

To create an evaluation framework for wearable devices design, it is important to determine the goal of evaluation, and the important factors to evaluate and the suitable evaluation tools to use. A number of studies have been working on product design or interface evaluation including usability evaluation, UX evaluation framework or integration of both. Evaluation framework of UX and usability framework, two general and prevailing frameworks in design and interface interaction evaluation, were proposed to assess whether the product can fully satisfy the user requirements in a particular context of use. Usability evaluation framework of ISO 9241-11 includes "Intended Goals, Context of Use, and Usability Measures" (Santos et al., 2015). When a product or service is used by a user under the context of use and intended goals, usability is measured by effectiveness, efficiency, and satisfaction. UX is evaluated by subjective measures as with satisfaction of usability. Evaluation framework of user experience, based on ISO 9241-11 and ACSI, includes Intended Goals, Context of Use, and UX Measures. In the UX Measure component, each component is supposed to be broken down into subindices (sub-questions) such as "Perceived Quality, Perceived Value, Complaints and Loyalty".

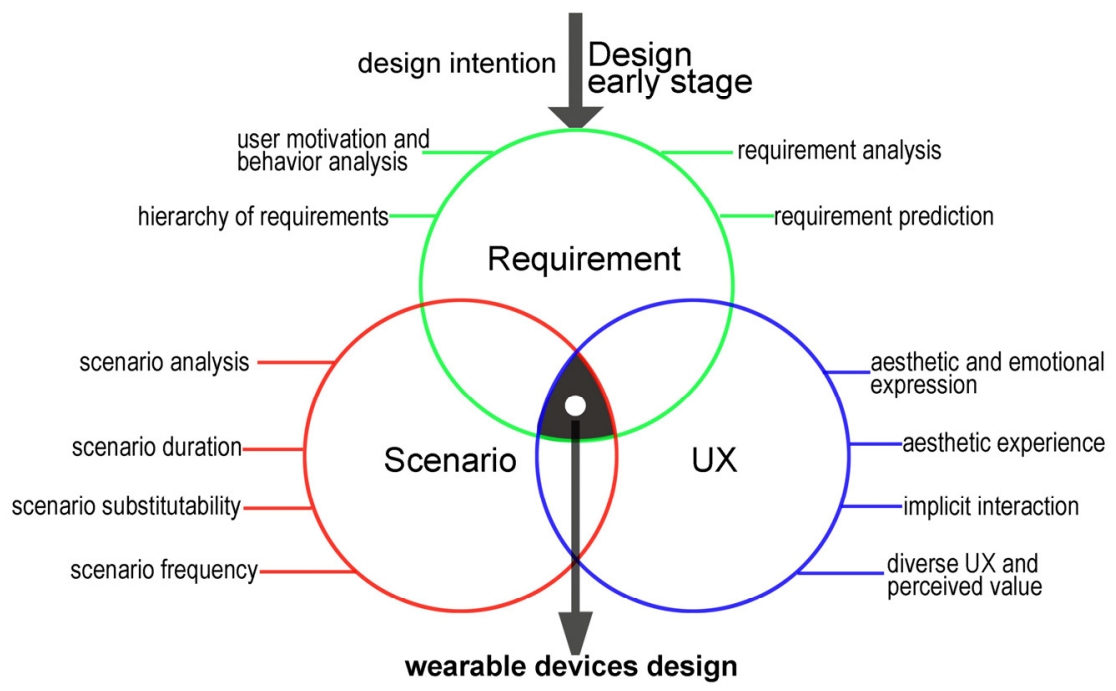

Figure 3. RSEF of wearable devices design evaluation 
However, UX and usability evaluation frameworks pay more attention on usability and user goal of human-product interaction, or user context, but ignore the importance of relations among scenariorequirement-behaviour-experience and lose sight of requirement analysis and influences from different kinds of user scenario with different dimensions, what makes it hard for designers to macroscopically and comprehensively understand the design intention in early stage of design development. The purpose of this framework is to provide guidance to designer and decision maker on wearable devices smart product design, related to evaluate the user requirement and UX in the specific scenario, using three dimensions impact matrix method. The proposed framework of this study is designed to be generic and suitable for different purposes and allow multiple criteria to be evaluated.

Our goal was to design a holistic and easy-to-use evaluation framework for wearable devices design and then assess, test, and validate it with case application to determine its usefulness. Thus our goal was not to design a framework to evaluate business outcomes, but to design a framework for evaluating the extent to which RSEF best practices are successful and support design processes and business decision making. This implies that there are parameters, as shown in Figure 3, determine design practice as well as decision making in wearable devices design. Meanwhile, RSEF is configured flexibility, possesses very good utility and expansibility, and enhances the accuracy of company leaders' decision making and the validity of design.

\subsection{Introducing Requirement-Scenario-Experience Framework (RSEF)}

The reasonable evaluation of wearable design must be user centered and take full account of potential business prospects. We should give full consideration to the systematicness, gradation and comprehensiveness of the index system construction process, and establish a scientific and reasonable evaluation index system for wearable product design. Combining the design features of wearable products and the criteria for usability assessment, the elements affecting the wearable design were systematically collected through a combination of interviews of user with wearable products and questionnaires. A total of 45 online questionnaires were obtained within two weeks and 42 valid questionnaires were remained. Through the analysis of the validity, reliability, and correlation of the questionnaire, a total of 3 criteria for wearable devices design evaluation, including user requirement, user scenario, and user experience, were constructed. Each evaluation criterion has relevant subdivision impact factors.

The RSEF of wearable devices design evaluation consists of three criteria. The first, the 'user requirement' describes the wearable devices design should meet at least one rigid requirement which is going to be utilized for the high priority to ensuring and improving the people's wellbeing. We concluded five impact factors of user requirement with different score to evaluate the extent of wearable and fashionable products to meet the requirements of users, shown in Table 1. The total score of requirement satisfaction is the sum of the five parts' score.

Table 1. User requirement impact factors

\begin{tabular}{ll}
\hline \multicolumn{1}{c}{ User requirement } & \multicolumn{1}{c}{ Impact factors } \\
\hline Time & service time; upgrade cycles; product delivery time \\
\hline Quality & Product performance; reliability; durability; maintenance \\
\hline Service & brand and product service; supply chain and after-sale service \\
\hline Design aesthetics & fashion trend; beauty of form \\
\hline Perceived value & emotion value; cost; mental expectation \\
\hline
\end{tabular}

The second component, 'user scenario', describes the scenario where the wearable smart products have been used with high frequency, long duration and irreplaceable property. Depending on the scenario analysis and evaluation process, the product should be designed to fit it. As shown in Table 2, different factors affect the frequency, duration and irreplaceable property. 
Table 2. User scenario impact factors

\begin{tabular}{ll}
\hline \multicolumn{1}{c}{ User scenario } & \multicolumn{1}{c}{ Impact factors } \\
\hline Frequency & use frequency; influence frequency; interaction frequency \\
\hline Duration & use duration; influence duration \\
\hline Non substitutability & particularity; meaning of existence \\
\hline
\end{tabular}

The 'UX factors' about aesthetics and emotional experience which become very important factors to decide whether to buy the products for consumers. In wearable smart product design, material, user interface, implicit interaction, lighting and other decoration are significant design expressions relating to UX, which can cause emotion changes of human product interaction.

Table 3. UX impact factors

\begin{tabular}{ll}
\hline \multicolumn{1}{c}{$U X$} & \multicolumn{1}{c}{ Impact factors } \\
\hline Human product interaction & functional experience; feeling of texture and material \\
\hline Human software interaction & Software interface; reliability; complexity \\
\hline Emotion and aesthetic experience & pleasure; satisfaction; form beauty \\
\hline Usability & battery charging times; software and product usability \\
\hline
\end{tabular}

The three components concern design intention in early stage and cognitive interpretation of the wearable products. The effectiveness and reliability of wearable product design benefit the performance of the product to satisfy the three parts of the RSEF. Figure 3 presents a whole evaluation perspective for wearable devices design. The user requirement part is the basic of the framework, if the requirements that the wearable devices create are not rigid needs, the product design might be into market even with perfect user experience and high frequency and long duration user scenario. Similarly, just like the "barrel principle", the occurrence of "minimum" in any part of this framework will directly affect the user adoption of wearable devices, even to be a decisive factor to low user adoption.

\subsection{A case study of RSEF framework}

Smart wristband are typical wearable form of smart intelligent hardware, Three smart wristband including Mi Band, Misfit shine and SONY Smart Band SWR10, as shown in Figure 4, were chosen in this study. We invited five designers with more than 10 years' experience and five wearable device users to participate in the evaluation work for the 3 products using RSEF. In evaluating the smart wristband, the ten participants should record every feeling of using the products in the process of product experiencing and submit a report related to requirements analysis, experience and usage scenario. According to the RSEF, the score of user requirement, user scenario and user experience were recorded. As shown in Table 4, the satisfaction degree of three components of wearable devices were scored as 10. The score of influence factors of the three parts were determined by the classification and analysis of the reports results.

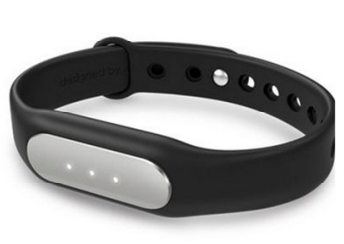

Mi Band

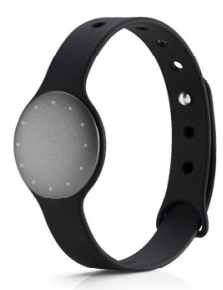

Misfit shine

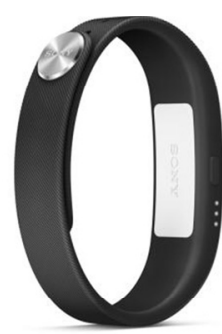

SONY SmartBand

Figure 4. Three smart wristband changed to be evaluated 
As shown in Table 4, the total score of user requirement satisfaction in Mi Band, Misfit shine and SONY Smart Band are 7.5, 8 and 4, it means that the participants believed the Mi Band and Misfit have the potential to gain a relative high satisfaction in the market than SONY Smart Band. The perceived value score of SONY Smart Band is not up to the half of the total points, it is possible a risk for SONY Smart Band to lose users in the future. The total score of User scenario satisfaction in Mi Band, Misfit shine and SONY Smart Band are 7.5, 4 and 1, it means that the 5 designers and 5 users believed the Mi Band can gain a relative higher satisfaction in the market than Misfit shine and SONY Smart Band. The frequency, duration score of Mi Band is obviously higher than Misfit shine and SONY Smart Band. From the result of nonsubstitutability, we can assume that users are likely to change their devices if there are better products into the market. The total score of User experience satisfaction are 7,7 and 4.5, the result show that the users get a better product experience from $\mathrm{Mi}$ Band and Misfit shine.

Table 4. Score of the three wearable devices

\begin{tabular}{|c|c|c|c|c|}
\hline \multicolumn{2}{|r|}{ RSEF } & \multirow{2}{*}{$\frac{\text { Mi Band }}{1.5}$} & \multirow{2}{*}{$\begin{array}{c}\text { Misfit shine } \\
1 \\
\end{array}$} & SONY Smart Band \\
\hline \multirow{5}{*}{$\begin{array}{c}\text { User } \\
\text { requirement } \\
\text { (10) }\end{array}$} & Time(2) & & & 0.5 \\
\hline & Quality(2) & 1.5 & 2 & 1.5 \\
\hline & Service(2) & 1 & 1.5 & 1 \\
\hline & Design aesthetics(2) & 1.5 & 2 & 0.5 \\
\hline & Perceived value(2) & 2 & 1.5 & 0.5 \\
\hline \multirow{3}{*}{$\begin{array}{c}\text { User } \\
\text { scenario } \\
(10)\end{array}$} & frequency(4) & 3.5 & 2 & 0.5 \\
\hline & duration(4) & 3.5 & 1.5 & 0.5 \\
\hline & Non substitutability(2) & 0.5 & 0.5 & 0 \\
\hline \multirow{4}{*}{$\begin{array}{c}\text { User } \\
\text { experience } \\
(10)\end{array}$} & Human product interaction(2) & 1.5 & 1 & 1.5 \\
\hline & Human software interaction(3) & 2 & 2 & 1 \\
\hline & Emotion and aesthetic experience(2) & 1 & 2 & 0.5 \\
\hline & Usability(3) & 2.5 & 2 & 1.5 \\
\hline \multicolumn{2}{|r|}{ Total score } & 22 & 19 & 9.5 \\
\hline
\end{tabular}

By comparing the RSEF evaluation results of three wearable devices, we can clearly identify the design risk of the smart product in a specific impact indicators. Furthermore, in the early stage of product design, RSEF method provided designers with a new understanding of the product being developed and gave guidance and reference to improve the success rate of new product development.

\section{Conclusions and further work}

There is an inevitable trend of intelligent and digital and continued interest in wearable, smart and fashionable industry, a whole new and complex area, making designers face more design constraints and decision-making trade-offs than ever before. This study was conducted in order to better understand the impact factors of wearable product design and evaluate the potential risk of fashion and smart product design in early design stage. We identified the attributes, characteristics and user behaviour of wearable and fashionable product and proposed a RSEF evaluation framework that includes these factors and their associated scales using a three-dimension impact matrix approach. Through the case study of the users and designers' evaluation of three wearable devices, we discovered that the framework can be utilized to evaluate the potential risk and chances of success, even though the three product have already in mass production, the RSEF evaluation framework have a great reference value in wearable product design process.

This research has two main contributions. The first is the holistic view of the wearable devices design, not limited to single parts such as product form, design aesthetics or user experience of such products. We summed up the factors and attributes of smart wearable product design from mental and cognitive behaviour, user motivation, implicit requirement and interaction to user scenario, user experience and 
usability. We analysed the relations between design intention and cognitive interpretation and presented the attributes of aesthetic experience and emotional expression in wearable product. Secondly, we revealed that the RSEF evaluation framework was generic and suitable for different purposes and allow multiple criteria to be evaluated in the smart wearable product design. The framework can be used for determining design directions in the concept phrase of development and for facilitating making decision in the scheme selection stage. It increased the success chance of product development and give guidance for designers to better understand the attributes of smart wearable product.

The further study we will focus on the concrete steps and quantitative methods with sufficient case application of RSEF and explore how wearable devices reshape user behaviour and cognition in the future.

\section{Acknowledgment}

This research is partly supported by National Key Technology R\&D Program, China (Grant No. 2015BAH21F01), 111 Project of China (Grant No.B13044). We would like to give special thanks to the designers who were willing to participate in this study.

\section{References}

Ananthanarayan, S. and Siek, K.A. (2012), "Persuasive wearable technology design for health and wellness", Proceedings of the 6th International Conference on Pervasive Computing Technologies for Healthcare and Workshops, San Diego, CA, USA, May 21-24, 2012, pp. 236-240. https://doi.org/10.4108/icst.pervasivehealth.2012.248694

Bødker, S. (2000), "Scenario in user-centred design-setting the stage for reflection and action", Interacting with Computers, Vol. 13 No. 1, pp. 61-75. https://doi.org/10.1016/S0953-5438(00)00024-2

Cao, Z., Gao, H., Qu, X., Yang, M. and Yang, X. (2013), "Fashion, Cooperation, and Social Interactions", PLoS ONE, Vol. 8 No. 1, pp. 1-21. https://doi.org/10.1371/journal.pone.0049441

Carroll, J.M. (2002), "Scenarios and design cognition", Proceedings of the IEEE International Conference on Requirements Engineering, Essen, Germany, September 9-13, 2002, pp. 3-5. https://doi.org/10.1109/ICRE.2002.1048498

Chu, M. (2009), "Natural and implicit interaction systems", Computer, Vol. 42 No. 8, pp. 109-111. https://doi.org/10.1109/MC.2009.264

Chung, K.-Y., Na, Y.-J. and Lee, J.-H. (2013), "Interactive design recommendation using sensor based smart wear and weather WebBot", Wireless Personal Communications, Vol. 73 No. 2, pp. 243-256. https://doi.org/10.1007/s11277-013-1234-5

Da Silva, O., Crilly, N. and Hekkert, P. (2015), "How people's appreciation of products is affected by their knowledge of the designers' intentions", International Journal of Design, Vol. 9 No. 2, pp. 21-33.

Davis, D.N. (2010), “Cognitive Architectures for Affect and Motivation”, Cognitive Computation, Vol. 2 No. 3 , pp. 199-216. https://doi.org/10.1007/s12559-010-9053-4

Deckers, E., Wensveen, S., Ahn, R. and Overbeeke, K. (2011), "Designing for perceptual crossing to improve user involvement", Proceedings of the SIGCHI Conference on Human Factors in Computing Systems (CHI '11), Vancouver, BC, Canada, May 7-12, 2011, pp. 1929-1938. https://doi.org/10.1145/1978942.1979222

Hallnäs, L. and Redström, J. (2001), "Slow technology - designing for reflection", Personal and Ubiquitous Computing, Vol. 5 No. 3, pp. 201-212. https://doi.org/10.1007/PL00000019

Kobayashi, H., Ueoka, R. and Hirose, M. (2009), "Wearable Forest Clothing System: Beyond Human-Computer Interaction", Leonardo, Vol. 42 No. 4, pp. 300-306. https://doi.org/10.1162/leon.2009.42.4.300

Norman, D.A. (2010), "Natural user interfaces are not natural”, Interactions, Vol. 17 No. 3, pp. 6-10. https://doi.org/10.1145/1744161.1744163

Santos, B.S., Ferreira, B.Q. and Dias, P. (2015), "Heuristic Evaluation in Information Visualization Using Three Sets of Heuristics: An Exploratory Study", Proceedings of the 17th International Conference on HumanComputer Interaction (HCI 2015), Los Angeles, CA, USA, August 2-7, 2015, pp. 259-270. https://doi.org/10.1007/978-3-319-20901-2_24

Sohn, M. and Nam, T.-J. (2015), "Understanding the attributes of product intervention for the promotion of proenvironmental behavior: A framework and its effect on immediate user reactions", International Journal of Design, Vol. 9 No. 2, pp. 55-77.

Toffler, A. (1970), Future shock, Random House, New York.

Wang, Y. and Yu, S. (2016), "Design for dynamic requirement and diverse user experience", Proceedings of the DESIGN 2016 / 14th International Design Conference, Dubrovnik, Croatia, May 16-19, 2016, pp. 553-560. 
Wang, Y. and Yu, S. (2017), "Car styling design decision-making model based on multi-objective particle swarm optimization”, Jisuanji Jicheng Zhizao Xitong / Computer Integrated Manufacturing Systems, Vol. 23 No. 4. https://doi.org/10.13196/j.cims.2017.04.001

Wang, Y., Yu, S. and Xu, T. (2017), "A user requirement driven framework for collaborative design knowledge management", Advanced Engineering Informatics, Vol. 33, pp. 16-28. https://doi.org/10.1016/j.aei.2017.04.002

Wei, W., Xiaodan, H., Jijun, Z. and Yanguang, S. (2014), “Implicit Human- Computer Interaction”, Information and Control, Vol. 2014 No. 1.

Woodruff, R.B. (1997), "Customer value: The next source for competitive advantage", Journal of the Academy of Marketing Science, Vol. 25 No. 2, pp. 139-153. https://doi.org/10.1007/BF02894350

Xenakis, I. and Arnellos, A. (2013), "The relation between interaction aesthetics and affordances", Design Studies, Vol. 34 No. 1, pp. 57-73. https://doi.org/10.1016/j.destud.2012.05.004

Yoon, H., Park, S.-H. and Lee, K.-T. (2016), "Lightful user interaction on smart wearables", Personal and Ubiquitous Computing, Vol. 20 No. 6, pp. 973-984. https://doi.org/10.1007/s00779-016-0959-Z

Zhou, E.-G., Zhou, J. and Li, B.-B. (2011), "Usability Evaluation Factors Research in Network Database System", Proceedings of the 4th International Conference on Internationalization, Design and Global Development (IDGD 2011), Orlando, FL, USA, July 9-14, 2011, pp. 283-290. https://doi.org/10.1007/978-3642-21660-2_32

Dr. Yahui Wang

Northwestern Polytechnical University, Shaanxi Engineering Laboratory for Industrial Design

No. 127, Youyi Road (West), Beilin, Xi'an City, Shaanxi Province, PRC, 710072 Xi'an, China

Email: yhwangmn@163.com 